\title{
Operation? Wie hätten Sie's denn gerne?
}

B etrachtet man die Angebote operativer Leistungen deutscher Kliniken, ist das Spektrum fast überall durch laparoskopische Operationen geprägt. Damit entsteht durchaus der Eindruck, hier muss möglichst viel und zum Teil auch „Ausgefallenes“ angeboten werden, um sich als die Klinik darzustellen, die man aufsuchen sollte. Überall wird nur noch „geschont“ und „ohne“ Schnitte durch das Schlüsselloch minimalisiert. Sogar Begriffe wie Mikrochirurgie und Minimalchirurgie sind zu lesen!

Wer das als Laie sieht, muss doch richtig Lust auf eine derartige Operation bekommen. Zum Teil ist das auch so gewollt und nichts anderes, als die Antwort auf den dringenden Wunsch der Verwalter und der Geschäftsführer der Kliniken, unverhohlen Reklame zu machen. Allerdings ist dies nur der Versuch, dem politisch erzeugten Druck im Kampf um das Überleben einer Klinik entgegenzuwirken bzw. diesen umzulenken.

Auf keinen Fall dürfen Weiterentwicklungen und damit der Fortschritt, zu dem natürlich auch die chirurgisch-endoskopischen Operationsmethoden gehören, gebremst oder gar blockiert werden. Selbstverständlich ist gut, dass heute beispielsweise eine unkomplizierte Operation an der Gallenblase fast überall völlig unaufgeregt als laparoskopischer Routineeingriff erfolgen kann. Doch schon bei der Behandlung einer Leistenhernie kommt das Problem verschiedener, konkurrierender Verfahren hinzu. Welches ist das Beste? Das weiß keiner ganz genau, obwohl die Operation über 20 000-mal jährlich in Deutschland durchgeführt wird. Die endoskopische Fundoplicatio wird ebenfalls mit breitester Indikationsstellung - im Gegensatz zu früher - mit verschiedensten Verfahrensweisen durchgeführt. Eine wissenschaftlich fundierte Prüfung der endoskopischen Operationen erfolgte vor der Einführung jedoch nicht und steht auch jetzt noch aus! Man stelle sich das zum Beispiel für die Einführung von Medikamenten vor - undenkbar!

Zwar gibt es in Deutschland verschiedenste Studien zu den gängigen endoskopischen Operationserfahren, koordiniert werden diese aber erst jetzt, beispielsweise vom Klinischen Studienzentrum Chirurgie in Heidelberg unter der Leitung von Prof. Dr. M. Büchler. Warum dieser Hinweis?

Wenn endoskopische Verfahrensweisen als Qualitätssteigerungen verkauft und mit ihnen Reklame gemacht werden muss - wovon wiederum teilweise sogar das Überleben von Kliniken abzuhängen scheint -, dann sollte man auch die Gefahren bedenken, die solche Zwänge mit sich bringen. Jede Klinik muss diese Verfahren möglichst umfangreich oder mit Besonderheiten an- bieten, um bestehen zu können. Und eben nicht nur die Verfahren die schon Routine sind, sondern möglichst auch solche, die noch Aufmerksamkeit erregen, auch wenn sie nur ein paar mal im Jahr durchgeführt werden.

Routine?! - Wer kann oder würde da schon auf die abdominalen endoskopischen Operationen bei malignen Erkrankungen verzichten? Sie sind ja allzu oft schon als so gut machbar publiziert und hochstilisiert worden. Oder auch bei Morbus Crohn? Ohne auf Einzelheiten einzugehen, ist dieser Eingriff sicher zum Teil für sehr Versierte, aber eben auch nur für diese machbar! Aber Vorsicht, nicht alles, was technisch machbar ist, kann man auch guten Gewissens empfehlen!

Gerade die neuen und deshalb als „modern“ bezeichneten Verfahren geben Anlass zu dem Hinweis, dass sich Ärzte zwar intensiv mit den geschäftlichen Belangen ihres Krankenhauses auseinandersetzen und unbedingt positiv für diese einsetzen sollten. Niemand darf aber dazu verführt werden oder sich verführen lassen, die eigenen erlernten Indikationsstellungen und für sich erprobten Routineverfahrensweisen aus ausschließlich kommerziellem Interesse aufs Spiel zu setzen. Die Politik würde das wohl wenig stören. Unsere Patienten würde es brennend interessieren!

Wir brauchen wissenschaftlich abgesicherte Ergebnisse - auch bei laparoskopischen und endoskopischen Verfahrensweisen -, an denen sich jeder orientieren kann. Die Federführung dabei dürfen nicht private Institute erhalten, die sicher auch viel Aufbauarbeit mitgeleistet haben, sondern eindeutig die medizinisch wissenschaftlichen Fachgesellschaften.

Die heute offen und ohne Scheu von den Ökonomen geforderte Reklame - häufig mit dem seriös anmutenden Terminus „Benchmarking“ verharmlost - steht in drastischer Konkurrenz zum ärztlichen Ethos! Wie hätten Sie's denn gerne?

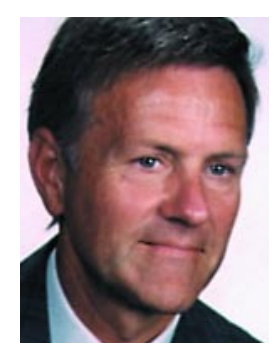

Prof. Dr. Burckart Stegemann, Hagen

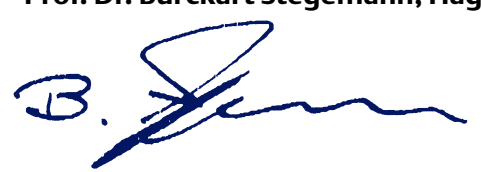

